PROCEEDINGS OF THE

AMERICAN MATHEMATICAL SOCIETY

Volume 131, Number 3, Pages 719-724

S 0002-9939(02)06649-2

Article electronically published on July 25, 2002

\title{
A $q$-SERIES IDENTITY AND THE ARITHMETIC OF HURWITZ ZETA FUNCTIONS
}

\author{
GWYNNETH H. COOGAN AND KEN ONO
}

(Communicated by David E. Rohrlich)

\begin{abstract}
Using a single variable theta identity, which is similar to the Jacobi Triple Product identity, we produce the generating functions for values of certain expressions of Hurwitz zeta functions at non-positive integers.
\end{abstract}

\section{Introduction AND STATEMENT OF RESULTS}

In a recent paper [3], D. Zagier used a $q$-series identity to prove that

$$
-e^{-t / 24} \sum_{n=0}^{\infty}\left(1-e^{-t}\right)\left(1-e^{-2 t}\right) \cdots\left(1-e^{-n t}\right)=\frac{1}{2} \sum_{n=0}^{\infty} L\left(\chi_{12},-2 n-1\right) \frac{(-t / 24)^{n}}{n !}
$$

where $\chi_{12}$ is the Kronecker character for $\mathbb{Q}(\sqrt{12})$. Subsequently, the second author, G. E. Andrews and J. Jimenez Urroz [1] obtained two infinite families of such $q$ series identities. In a few ad hoc cases, these identities produced further generating functions for $L$-function and zeta values such as

$-e^{-t / 8} \sum_{n=0}^{\infty} \frac{\left(1-e^{-2 t}\right)\left(1-e^{-4 t}\right) \cdots\left(1-e^{-2 n t}\right)}{\left(1+e^{-t}\right)\left(1+e^{-3 t}\right) \cdots\left(1+e^{-(2 n+1) t}\right)}=\frac{1}{2} \sum_{n=0}^{\infty} L\left(\chi_{2},-2 n-1\right) \cdot \frac{(-t / 8)^{n}}{n !}$.

Here $\chi_{2}$ is the Kronecker character for $\mathbb{Q}(\sqrt{2})$.

Since these results are born out of the arithmetic of basic hypergeometric functions (for more on basic hypergeometric functions, see [2]), we refer to them as 'hypergeometric'. Clearly, this method for computing $\zeta$-values at non-positive integral arguments is very different from the classical approach involving special values of Bernoulli polynomials. Here we show that such hypergeometric generating functions are widespread, and may be obtained in a systematic, rather than ad hoc, fashion.

We begin by recalling the Jacobi Triple Product Identity [2, p. 19, eq. (17.3)]

$$
\prod_{n=1}^{\infty}\left(1-q^{2 n}\right)\left(1+z^{2} q^{2 n-1}\right)\left(1+z^{-2} q^{2 n-1}\right)=\sum_{n=-\infty}^{\infty} z^{2 n} q^{n^{2}}
$$

Received by the editors October 22, 2001.

2000 Mathematics Subject Classification. Primary 11B65, 11M35.

The authors thank the National Science Foundation for their generous support. The second author also thanks the Alfred P. Sloan Foundation and the David and Lucile Packard Foundation for their generous support. 
Our results depend on a hypergeometric analog of this classical result. We begin by fixing notation. Let $(a ; q)_{0}:=1$, and for every positive integer $n$ let

$$
(a ; q)_{n}:=(1-a)(1-a q)\left(1-a q^{2}\right) \cdots\left(1-a q^{n-1}\right) .
$$

Proposition 1.1. The following formal power series identity is true:

$$
A(z ; q):=\frac{1}{1+z} \cdot \sum_{n=0}^{\infty} \frac{(z ; q)_{n} z^{n}}{(-z q ; q)_{n}}=\sum_{n=0}^{\infty}(-1)^{n} z^{2 n} q^{n^{2}} .
$$

This identity follows from an identity due to Rogers and Fine [2, p. 15, eq. (14.1)]. We include a proof in the next section for the benefit of those unfamiliar with basic hypergeometric series. The following is the result of a simple calculation.

Corollary 1.2. If $0 \leq b<a$ and $\zeta_{z}$ and $\zeta_{q}$ are complex numbers, then

$$
\begin{aligned}
\Theta\left(a, b, \zeta_{z}, \zeta_{q} ; z, q\right) & :=Z^{b} Q^{b^{2}} \cdot A\left(Z^{a / 2} Q^{a b} ; Q^{a^{2}}\right) \\
& =\sum_{n=0}^{\infty}(-1)^{n} \zeta_{z}^{a n+b} \zeta_{q}^{(a n+b)^{2}} z^{(a n+b)} q^{(a n+b)^{2}}
\end{aligned}
$$

where $Z:=\zeta_{z} z$ and $Q:=\zeta_{q} q$.

To illustrate our results regarding hypergeometric generating functions for $\zeta$ values, consider the following series:

$$
\Theta(2,1,1,1 ; z, q):=\frac{z q}{1+z q^{2}} \cdot \sum_{n=0}^{\infty} \frac{\left(z q^{2} ; q^{4}\right)_{n} z^{n} q^{2 n}}{\left(-z q^{6} ; q^{4}\right)_{n}}=\sum_{n=0}^{\infty}(-1)^{n} z^{(2 n+1)} q^{(2 n+1)^{2}} .
$$

Since the Taylor series of $1-e^{-n t}$ is

$$
1-e^{-n t}=t^{n}-\frac{t^{2 n}}{2}+\frac{t^{3 n}}{6}-\cdots
$$

we find that $\Theta\left(2,1,1,1 ; 1, e^{-t}\right)$ is a well defined formal power series in $t$ :

$$
\Theta\left(2,1,1,1 ; 1, e^{-t}\right)=\frac{e^{-t}}{1+e^{-2 t}} \cdot \sum_{n=0}^{\infty} \frac{\left(e^{-2 t} ; e^{-4 t}\right)_{n} e^{-2 n t}}{\left(-e^{-6 t} ; e^{-4 t}\right)_{n}}=\frac{1}{2}+\frac{1}{2} t+\frac{5}{4} t^{2}+\cdots
$$

Observe that the naive substitution of $z=1$ and $q=e^{-t}$ in the right-hand side of (1.2) does not produce a well defined $t$-series.

It turns out that the series in (1.3) satisfies

$$
\Theta\left(2,1,1,1 ; 1, e^{-t}\right)=\frac{1}{2}+\frac{1}{2} t+\cdots=\sum_{n=0}^{\infty} L\left(\chi_{-1},-2 n\right) \cdot \frac{(-t)^{n}}{n !} .
$$

Here $\chi_{-1}$ is the non-trivial Dirichlet character with modulus 4. Therefore, (1.4) is a hypergeometric generating function whose coefficients interpolate values of $L\left(\chi_{-1}, s\right)$ at non-positive even integers.

If $0<c \leq 1$ is a rational number, then let $\zeta(s, c)$ denote the Hurwitz zetafunction

$$
\zeta(s, c):=\sum_{n=0}^{\infty} \frac{1}{(n+c)^{s}} .
$$


These functions possess an analytic continuation to the whole complex plane, with the exception of a simple pole at $s=1$. It is easy to see that (1.4) is equivalent to

$$
\Theta\left(2,1,1,1 ; 1, e^{-t}\right)=\sum_{n=0}^{\infty}(\zeta(-2 n, 1 / 4)-\zeta(-2 n, 3 / 4)) \cdot \frac{(-16 t)^{n}}{n !} .
$$

The preceding example illustrates our first general result.

Theorem 1.3. Suppose that $0 \leq b<a$ are integers, and suppose that $\alpha, \beta$ and $m$ are positive integers for which both

1) $\operatorname{ord}_{2}(m)<\operatorname{ord}_{2}\left(2 a^{2} \beta\right)$,

2) $a \alpha+2 a \beta(b+a k) \equiv 0(\bmod 2 m)$ for some residue class $k(\bmod 2 m)$.

If $\zeta_{z}:=e^{2 \pi i \alpha / m}$ and $\zeta_{q}:=e^{2 \pi i \beta / m}$, then as a formal power series in $t$ we have

$$
\begin{aligned}
& \Theta\left(a, b, \zeta_{z}, \zeta_{q} ; 1, e^{-t}\right) \\
& \quad=\sum_{n=0}^{\infty}\left(\sum_{j=0}^{m-1} c_{j} \zeta\left(-2 n, \frac{2 a j+b}{2 a m}\right)-d_{j} \zeta\left(-2 n, \frac{(2 j+1) a+b}{2 a m}\right)\right) \frac{\left(-4 a^{2} m^{2} t\right)^{n}}{n !},
\end{aligned}
$$

where $c_{j}:=\zeta_{z}^{(2 j a+b)} \zeta_{q}^{(2 j a+b)^{2}}$ and $d_{j}:=\zeta_{z}^{((2 j+1) a+b)} \zeta_{q}^{((2 j+1) a+b)^{2}}$.

Here we highlight one special case of Theorem 1.3. The following corollary provides the generating function for the values at non-positive even integers for certain differences of Hurwitz zeta functions.

Corollary 1.4. If $0 \leq b<a$, then as a formal power series in $t$ we have

$$
\Theta\left(a, b, 1,1 ; 1, e^{-t}\right)=\sum_{n=0}^{\infty}\left(\zeta\left(-2 n, \frac{b}{2 a}\right)-\zeta\left(-2 n, \frac{a+b}{2 a}\right)\right) \frac{\left(-4 a^{2} t\right)^{n}}{n !}
$$

Note that (1.6) is a special case of this result.

We now obtain further such series using Corollary 1.2. To state these results, let $\Theta^{\prime}\left(a, b, \zeta_{z}, \zeta_{q} ; z, q\right)$ denote the series obtained by differentiating, summand by summand, $\Theta\left(a, b, \zeta_{z}, \zeta_{q} ; z, q\right)$ with respect to $z$.

Theorem 1.5. Assuming the notation and hypotheses in Theorem 1.3, as a formal power series in $t$ we have

$\frac{\Theta^{\prime}\left(a, b, \zeta_{z}, \zeta_{q} ; 1, e^{-t}\right)}{2 a m}$

$$
=\sum_{n=0}^{\infty} \sum_{j=0}^{m-1}\left(c_{j} \zeta\left(-2 n-1, \frac{2 j a+b}{2 a m}\right)-d_{j} \zeta\left(-2 n-1, \frac{(2 j+1) a+b}{2 a m}\right)\right) \frac{\left(-4 a^{2} m^{2} t\right)^{n}}{n !}
$$

where $c_{j}:=\zeta_{z}^{2 a j+b} \zeta_{q}^{(2 a j+b)^{2}}$ and $d_{j}:=\zeta_{z}^{((2 j+1) a+b)} \zeta_{q}^{((2 j+1) a+b)^{2}}$.

As a corollary, we have the following counterpart to Corollary 1.4.

Corollary 1.6. If $0 \leq b<a$, then as a formal power series in $t$ we have

$$
\frac{\Theta^{\prime}\left(a, b, 1,1 ; 1, e^{-t}\right)}{2 a}=\sum_{n=0}^{\infty}\left(\zeta\left(-2 n-1, \frac{b}{2 a}\right)-\zeta\left(-2 n-1, \frac{a+b}{2 a}\right)\right) \frac{\left(-4 a^{2} t\right)^{n}}{n !} .
$$




\section{Proof of Proposition 1.1}

We prove Proposition 1.1 by following the method described in [2, pp. 14-15, Sec. 13].

Proof of Proposition 1.1. Consider the function

$$
B(z, t ; q):=\sum_{n=0}^{\infty} \frac{(z ; q)_{n} t^{n}}{(-z q ; q)_{n}}=\sum_{n=0}^{\infty} B_{n} t^{n}
$$

Note that $A(z ; q)=\frac{1}{1+z} B(z, z ; q)$. We determine its transformation under the map

$$
B(z, t ; q) \rightarrow B(z q, t q ; q),
$$

and iterate it to obtain the proposition. The fact that $(z ; q)_{n}=(1-z)(z q ; q)_{n-1}$ implies

$$
B(z, t ; q)=1+\frac{(1-z)}{(1+z q)} t B(z q, t ; q) .
$$

Since $B_{n+1}\left(1+z q^{n+1}\right)=B_{n}\left(1-z q^{n}\right)$, by multiplying by $t^{n+1}$ and summing on $n$ we obtain

$$
\sum_{n=0}^{\infty} B_{n+1} t^{n+1}+z \sum_{n=0}^{\infty} B_{n+1}(t q)^{n+1}=t \sum_{n=0}^{\infty} B_{n} t^{n}-t z \sum_{n=0}^{\infty} B_{n}(t q)^{n} .
$$

This is equivalent to the second transformation formula

$$
B(z, t ; q)=\frac{(1+z)}{(1-t)}-z \frac{(1+t)}{(1-t)} B(z, t q ; q) .
$$

Transformations (2.2) and (2.3) imply

$$
B(z, t ; q)=\frac{(1-z t)}{(1-t)}-\frac{(1-z)(1+t)}{(1-t)(1+z q)} t q z B(z q, t q ; q) .
$$

One iteration of this transformation formula yields

$$
\begin{aligned}
B(z, t ; q)=\frac{(1-z t)}{(1-t)}- & \frac{(1-z)(1+t)\left(1-z t q^{2}\right)}{(1-t)(1+z q)(1-t q)} z t q \\
& +\frac{(1-z)(1-z q)(1+t)(1+t q)}{(1-t)(1-t q)(1+z q)\left(1+z q^{2}\right)} z^{2} t^{2} q^{4} B\left(z q^{2}, t q^{2} ; q\right) .
\end{aligned}
$$

An infinite number of iterations yields

$$
B(z, t ; q)=\frac{1}{1-t} \cdot \sum_{n=0}^{\infty} \frac{(z ; q)_{n}(-t ; q)_{n}}{(-z q ; q)_{n}(t q ; q)_{n}}\left(1-z t q^{2 n}\right)(-1)^{n} z^{n} t^{n} q^{n^{2}} .
$$

If $t=z$ above, the cancellation among the $q$-factorial terms above yields

$$
A(z ; q)=\frac{1}{1+z} \cdot B(z, z ; q)=\sum_{n=0}^{\infty}(-1)^{n} z^{2 n} q^{n^{2}} .
$$




\section{Proof of Theorems 1.3 And 1.5}

Proof of Theorems 1.3 and 1.5. We let $z=1$ and replace $q$ by $e^{-t}=1-t+t^{2} / 2+$ $\ldots$, and notice that the sum is a convergent power series in $t$ when $\left(1-\zeta_{z}^{a / 2} \zeta_{q}^{a b+k a^{2}}\right)$ vanishes for infinitely many integers $k$ and $\left(1+\zeta_{z}^{a / 2} \zeta_{q}^{a b+c a^{2}}\right)$ does not vanish for any integer $c$.

To guarantee that the numerators of the summands in this series start with larger and larger powers of $t$, we require that $\zeta_{z}^{a / 2} \zeta_{q}^{a b+k a^{2}}=1$ for infinitely many integers $k$. This implies that

$$
a \alpha+2 \beta\left(a b+k a^{2}\right) \equiv 0 \quad(\bmod 2 m)
$$

This is the second hypothesis in Theorem 1.3. Next, if both the numerator and the denominator vanish, then for some $k$ and $c=k+d \in \mathbb{Z}$

$$
\begin{aligned}
& 1-\zeta_{z}^{a / 2} \zeta_{q}^{a b} \zeta_{q}^{a^{2} k}=0 \text { and } 1+\zeta_{z}^{a / 2} \zeta_{q}^{a b} \zeta_{q}^{a^{2} c}=0 \\
\Rightarrow \quad & \zeta_{q}^{a^{2} k}+\zeta_{q}^{a^{2} c}=e^{\frac{2 \pi i a^{2} k \beta}{m}}\left(1+e^{2 \pi i \frac{a^{2} d \beta}{m}}\right)=0 \quad \Rightarrow \quad m=\frac{2 a^{2} d \beta}{2 n+1},
\end{aligned}
$$

for $d$ and some odd integer $2 n+1$. However, this is prohibited by the first hypothesis in Theorem 1.3. Therefore, the formal power series in $t$ is well defined.

Define coefficients $c(n)$ by

$$
\Theta\left(a, b, \zeta_{z}, \zeta_{q} ; 1, e^{-t}\right)=\sum_{n=0}^{\infty} c(n) t^{n}=\sum_{n=0}^{\infty}(-1)^{n} \zeta_{z}^{a n+b} \zeta_{q}^{(a n+b)^{2}} e^{-t(a n+b)^{2}} .
$$

We consider the Mellin transform of $\Theta\left(a, b, \zeta_{z}, \zeta_{q} ; 1, e^{-t}\right)$ :

$$
\begin{aligned}
\int_{0}^{\infty} \Theta\left(a, b, \zeta_{z}, \zeta_{q} ; 1, e^{-t}\right) t^{s-1} d t & =\int_{0}^{\infty} \sum_{n=0}^{\infty}(-1)^{n} \zeta_{z}^{a n+b} \zeta_{q}^{(a n+b)^{2}} e^{-t(a n+b)^{2}} t^{s-1} d t \\
& =\sum_{n=0}^{\infty}(-1)^{n} \zeta_{z}^{a n+b} \zeta_{q}^{(a n+b)^{2}} \int_{0}^{\infty} e^{-t(a n+b)^{2}} t^{s-1} d t \\
& =\sum_{n=0}^{\infty} \frac{(-1)^{n} \zeta_{z}^{a n+b} \zeta_{q}^{(a n+b)^{2}}}{(a n+b)^{2 s}} \int_{0}^{\infty} e^{-t} t^{s-1} d t \\
& =\Gamma(s) L(\psi, 2 s) .
\end{aligned}
$$

Here $L(\psi, s)$ is the generalized $L$-function defined by analytically continuing the Dirichlet series

$$
L(\psi, s):=\sum_{n=1}^{\infty} \frac{\psi(n)}{n^{s}}
$$

where $\psi(n):=(-1)^{(n-b) / a} \zeta_{z}^{n} \zeta_{q}^{n^{2}}$ if $n \equiv b(\bmod a)$, and 0 otherwise. Since $\psi$ has period $2 a m$ we can write $L(\psi, s)$ as a linear combination of Hurwitz zeta functions.

$$
L(\psi, s)=(2 a m)^{-s} \sum_{r=1}^{2 a m} \psi(r) \zeta\left(s, \frac{r}{2 a m}\right),
$$

which turns out as

$$
L(\psi, s)=(2 a m)^{-s} \sum_{j=0}^{m-1}\left(c_{j} \zeta\left(s, \frac{2 a j+b}{2 a m}\right)-d_{j} \zeta\left(s, \frac{a(2 j+1)+b}{2 a m}\right)\right),
$$


where

$$
c_{j}=\zeta_{z}^{2 a j+b} \zeta_{q}^{(2 a j+b)^{2}} \quad \text { and } \quad d_{j}=\zeta_{z}^{a(2 j+1)+b} \zeta_{q}^{(a(2 j+1)+b)^{2}} .
$$

On the other hand if we are careful about the way in which we evalute the integral in the Mellin transform, we are able to locate and identify its residues.

$$
\begin{aligned}
\int_{0}^{\infty} & \Theta\left(a, b, \zeta_{z}, \zeta_{q} ; 1, e^{-t}\right) t^{s-1} d t \\
& =\int_{0}^{1} \Theta\left(a, b, \zeta_{z}, \zeta_{q} ; 1, e^{-t}\right) t^{s-1} d t+\int_{1}^{\infty} \Theta\left(a, b, \zeta_{z}, \zeta_{q} ; 1, e^{-t}\right) t^{s-1} d t \\
& =\int_{0}^{1} \sum_{n=0}^{\infty} c(n) t^{n+s-1} d t+\int_{1}^{\infty} \Theta\left(a, b, \zeta_{z}, \zeta_{q} ; 1, e^{-t}\right) t^{s-1} d t \\
& =\int_{0}^{1} \sum_{n=0}^{N-1} c(n) t^{n+s-1}+O\left(t^{N+s-1}\right) d t+\int_{1}^{\infty} \Theta\left(a, b, \zeta_{z}, \zeta_{q} ; 1, e^{-t}\right) t^{s-1} d t \\
& =\sum_{n=0}^{N-1} \frac{c(n)}{s+n}+A(s),
\end{aligned}
$$

where because $e^{-t}$ is small for large $t, A(s)$ is analytic for $\operatorname{Re}(s)>-N$. It is clear now that the residue at $s=-n$ is $c(n)$. By (3.6), this implies that

$$
c(n)=\operatorname{Res}_{s=-n}(L(\psi, 2 s) \Gamma(s))=\frac{(-1)^{n}}{n !} \cdot L(\psi,-2 n)
$$

Therefore we find that

$$
\Theta\left(a, b, \zeta_{z}, \zeta_{q} ; 1, e^{-t}\right)=\sum_{n=0}^{\infty} \frac{(-1)^{n} L(\psi,-2 n) t^{n}}{n !} .
$$

Theorem 1.3 now follows from (3.3).

To prove Theorem 1.5, observe that the product rule implies that

$$
\Theta^{\prime}\left(a, b, \zeta_{z}, \zeta_{q} ; 1, e^{-t}\right)
$$

converges under the same hypotheses in Theorem 1.3. The only difference in the calculation of the $t$ expansion for the derivative is the factor $(a n+b)$ which ends up yielding $\Gamma(s) L(\psi, 2 s-1)$ in the analog of (3.6). The rest of the proof of Theorem 1.5 follows mutatis mutandis.

\section{REFERENCES}

[1] G.E. Andrews, J. Jimenez-Urroz, K. Ono, $q$-series identities and values of certain $L$-functions, Duke Math. J. 108 (2001), 395-419.

[2] N. Fine, Basic hypergeometric series and applications, Math. Surv. and Mono. 27, Amer. Math. Soc. 1988. MR 91j:33011

[3] D. Zagier, Vassiliev invariants and a strange identity related to the Dedekind eta-function, Topology 40 (2001), 945-960.

Department of Mathematics, University of Wisconsin, Madison, Wisconsin 53706

E-mail address: gwynneth@math.wisc.edu

Department of Mathematics, University of Wisconsin, Madison, Wisconsin 53706

E-mail address: ono@math.wisc.edu 